\title{
Impact of environmental moisture on tropical cyclone intensification
}

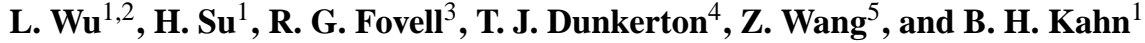 \\ ${ }^{1}$ Jet Propulsion Laboratory, California Institute of Technology, Pasadena, California, USA \\ ${ }^{2}$ Joint Institute for Regional Earth System Science and Engineering, University of California, Los Angeles, California, USA \\ ${ }^{3}$ University of California, Los Angeles, Los Angeles, California, USA \\ ${ }^{4}$ Northwest Research Associates, Inc., Bellevue, Washington, USA \\ ${ }^{5}$ University of Illinois at Urbana-Champaign, Urbana, Illinois, USA
}

Correspondence to: L. Wu (longtao.wu@jpl.nasa.gov)

Received: 9 April 2015 - Published in Atmos. Chem. Phys. Discuss.: 15 June 2015

Revised: 16 November 2015 - Accepted: 7 December 2015 - Published: 21 December 2015

\begin{abstract}
The impacts of environmental moisture on the intensification of a tropical cyclone (TC) are investigated in the Weather Research and Forecasting (WRF) model, with a focus on the azimuthal asymmetry of the moisture impacts relative to the storm path. A series of sensitivity experiments with varying moisture perturbations in the environment are conducted and the Marsupial Paradigm framework is employed to understand the different moisture impacts. We find that modification of environmental moisture has insignificant impacts on the storm in this case unless it leads to convective activity that deforms the quasi-Lagrangian boundary of the storm and changes the moisture transport into the storm. By facilitating convection and precipitation outside the storm, enhanced environmental moisture ahead of the northwestward-moving storm induces a dry air intrusion to the inner core and limits TC intensification. In contrast, increased moisture in the rear quadrants favors intensification by providing more moisture to the inner core and promoting storm symmetry, with primary contributions coming from moisture increase in the boundary layer. The different impacts of environmental moisture on TC intensification are governed by the relative locations of moisture perturbations and their interactions with the storm Lagrangian structure.
\end{abstract}

\section{Introduction}

While the forecast of tropical cyclone (TC) tracks has been significantly improved in the past several decades, the TC intensity forecast is still a great challenge for most operational numerical weather prediction centers (DeMaria et al., 2007).
Environmental moisture has been considered as one of the important factors for TC intensity forecasting. As one of the skillful predictors, the $850 \mathrm{hPa}$ relative humidity (RH) averaged between 200 and $800 \mathrm{~km}$ from storm center has been used routinely in the Statistical Hurricane Intensity Prediction Scheme (SHIPS) for hurricane intensity forecasting at the National Hurricane Center (Kaplan et al., 2010).

Theoretical and modeling studies have suggested high environmental moisture may be conducive to TC intensification (e.g., Emanuel et al., 2004; Kimball, 2006). Dry air intrusion could lead to a weakening of a TC by inducing asymmetric convective activity and/or transporting low equivalent potential temperature $\left(\theta_{\mathrm{e}}\right)$ air into the sub-cloud layer and storm inflow (e.g., Braun et al., 2012; Emanuel, 1989; Ge et al., 2013; Kimball, 2006; Tao and Zhang, 2014). However, some studies (e.g., Kimball 2006; Wang, 2009; Ying and Zhang, 2012) showed that substantial moisture may also cause a negative impact on TC strength by facilitating the formation of TC rainbands, which reduces the horizontal pressure gradient of a TC. In idealized simulations, Hill and Lackmann (2009) varied RH values in the moist envelope $100 \mathrm{~km}$ beyond the TC core and found that larger RH results in the establishment of wider TCs with more prominent outer rainbands. However, in their study, TC intensity was nearly insensitive to environmental $\mathrm{RH}$ despite the variation in rainband activity.

Braun et al. (2012) showed that dry air located $270 \mathrm{~km}$ away from the storm center had little impact on hurricane intensity with no mean flow. Dry air intrusion into the storm vortex, however, suppressed convective activity and increased the asymmetry of convection, leading to a weakening of the storm. While a dry air envelope had no significant 
impact on hurricane intensity, the storm size was reduced. Vertical shear can significantly enhance the suppression effect of dry air intrusion (Tang and Emanuel, 2012; Ge et al., 2013; Tao and Zhang, 2014). By modifying the diabatic heating rate due to cloud microphysical process, Wang (2009) demonstrated that diabatic cooling in the outer spiral rainbands helped the TC remain intense and compact. Increased latent heat release in the outer spiral rainbands decreased the intensity but increased the TC size. In a sensitivity study of Typhoon Talim (2005), Ying and Zhang (2012) showed that enhanced moisture promoted convection in outer rainbands and resulted in the weakening of the storm while dry air inhibited outer rainbands and contributed to a stronger storm with smaller size. The storm was more sensitive to the moisture perturbation residing to the north than to the south due to its shorter travel time into the storm vortex.

Composite studies using analyses data sets and satellite observations (Kaplan and DeMaria, 2003; Hendricks et al., 2010; Wu et al., 2012) have shown that rapid intensification (RI) of TCs is associated with higher environmental RH in the lower and middle troposphere than non-RI events. Using satellite observations, Shu and Wu (2009) showed that the dry Saharan air layer (SAL) can affect TC intensity in both favorable and unfavorable manners. TCs tend to intensify when dry SAL air is present in the northwest quadrant of TCs. However, TCs tend to weaken when dry air intrudes within $360 \mathrm{~km}$ of the TC center in the southwest and southeast quadrants. Substantial azimuthal asymmetry of RH is also found in TCs' environments based on 9 years of satellite observations, with rear quadrants (relative to storm motion) being moister than front quadrants, especially during RI (Wu et al., 2012).

Most previous modeling studies prescribed moisture perturbations without specifically considering their relative location to a storm vortex (e.g., in the environment, outer rainband or inner core, front or rear quadrants), which may cause different impacts on the storm structure and intensity. In this study, we investigate the impacts of environmental moisture on TC intensity and structure using the Weather Research and Forecasting (WRF) model with artificially modified environmental moisture surrounding a storm vortex. Guided by the observational composite study by Wu et al. (2012), we focus on the azimuthally asymmetric effects of environmental moisture in the front and rear quadrants. Section 2 provides the model description and experiment design. The Marsupial Paradigm framework (Dunkerton et al., 2009) is also introduced in Sect. 2 as a tool to interpret the moisture impacts on the storm. Section 3 describes the evolution of the simulated storm in the control experiment. The results from sensitivity experiments are presented in Sect. 4. The findings from this study are summarized in Sect. 5.

\section{WRF experiments and analysis framework}

\subsection{Model description}

To examine the role of environmental moisture on TC intensification, we drive the WRF model with initial and boundary conditions from a real-case hurricane, in particular Hurricane Earl (2010). Hurricane Earl originated from a tropical wave west of the Cape Verde Islands on 23 August 2010. It moved westward across the Atlantic and gradually strengthened to a tropical storm. Before the RI at 00:00 UTC on 29 August (Fig. 1a), a dry zone consisting of precipitable water vapor (PWV) less than $4.5 \mathrm{~cm}$ was located to the west of the storm, in the front quadrant relative to the storm propagation. Meanwhile, a broad moist region was observed to the south and southeast of the storm. Such a "dry front and moist rear" environmental moisture structure is typical of a rapidly intensifying hurricane as found in Wu et al. (2012). Earl underwent a RI from 06:00 UTC, 29 August, to 00:00 UTC, 31 August. The maximum wind speed (MWSP) increased by $31 \mathrm{~m} \mathrm{~s}^{-1}$ while the minimum sea level pressure (MSLP) deepened by $53 \mathrm{hPa}$ in $36 \mathrm{~h}$.

Inspired by the rapid intensification of Hurricane Earl (2010), we initialize the Advanced Research WRF model V3.3.1 (Skamarock et al., 2008) at 00:00 UTC, 29 August 2010, and run it for $48 \mathrm{~h}$. Simulations are conducted with a parent grid at $9 \mathrm{~km}$ horizontal resolution and a vortex-following nested grid at $3 \mathrm{~km}$ resolution. Experiments show that simulated results are not sensitive to the horizontal resolution of the parent grid with similar inner domains. There are 50 model levels in the vertical from the surface to $20 \mathrm{hPa}$, and the initial and boundary conditions were derived from the interim ECMWF (European Centre for Medium-Range Weather Forecasts) reanalysis (ERAInterim) (http://rda.ucar.edu/datasets/ds627.0/). For all the experiments, we employ the Thompson et al. (2008) microphysical scheme, the Rapid Radiative Transfer Model for GCMs (RRTMG) shortwave and longwave schemes (Iacono et al., 2008) and the Yonsei University planetary boundary layer scheme (Hong et al., 2006). The Kain-Fritsch cumulus scheme (Kain, 2004) is used in the parent domain while no cumulus scheme is used in the moving nested inner grids.

As the model is initialized solely from the coarseresolution reanalysis, the initial TC is weaker and less organized than the actual storm was and thus at least a portion of its subsequent intensification represents a response to the improved resolution. Our focus is on how environmental moisture perturbations directly and indirectly influence how the storm organizes subsequent to initialization. To assess potential impacts of the initial conditions, the WRF control (CTRL) simulation consists of five ensemble members with randomly generated RH perturbations of less than $1 \%$ added to the initial specific humidity field at all model horizontal and vertical grids. In the following discussions, the 

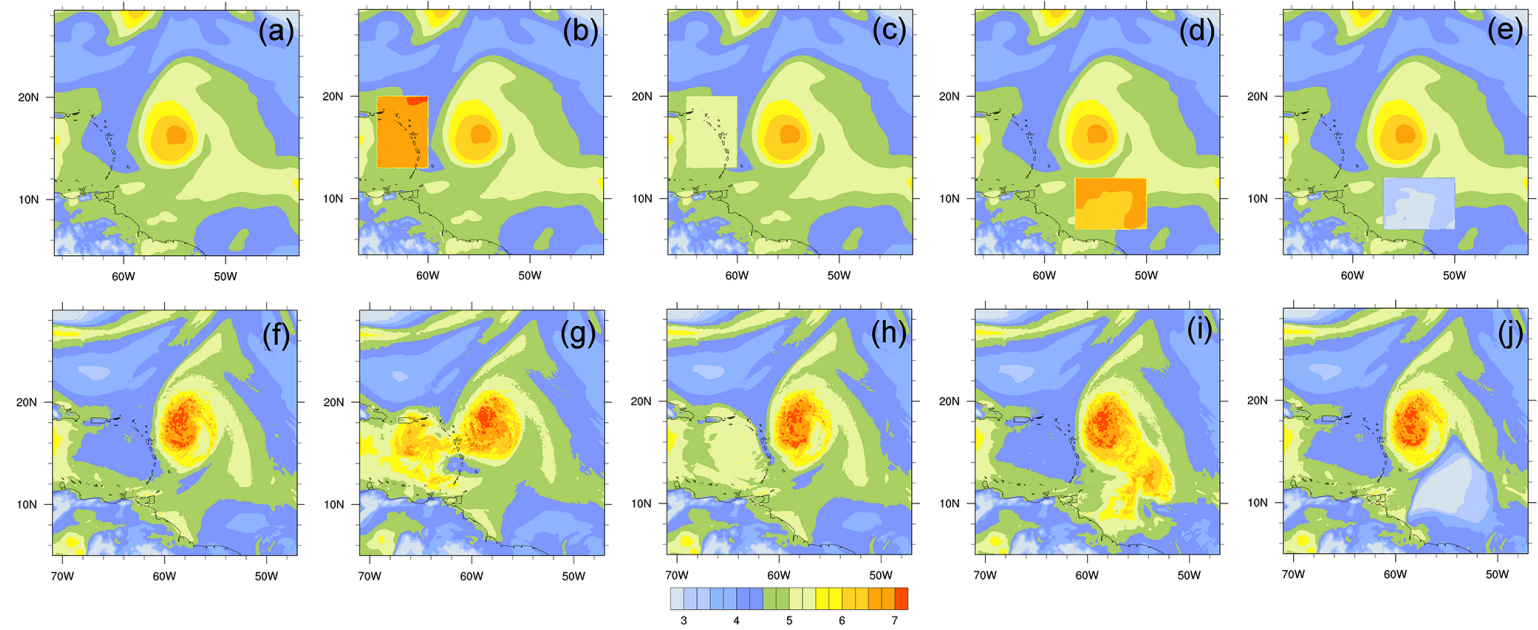

Figure 1. Column-integrated PWV (cm) at the initialization of the WRF simulations: (a) CTRL, (b) MF, (c) MFI, (d) MR and (e) DR; $12 \mathrm{~h}$ forecast for (f) CTRL, (g) MF, (h) MFI, (i) MR and (j) DR.

CTRL and other sensitivity experiments refer to the ensemble means of the respective five ensemble members.

\subsection{Experiment design}

The sensitivity experiments are conducted by placing moisture perturbations of varying magnitudes at different locations relative to the storm at the initial time (Fig. 1a-e). The zones are rectangular in shape and sharply bounded and, as a consequence, could serve as focal points for convective activity if conditions are sufficiently favorable. We explored tapering the edges of the moisture perturbations and found it did not materially alter our conclusions.

In the moist front (MF) experiment (Fig. 1b), an artificially moistened zone of $5^{\circ}$ in longitude and $7^{\circ}$ in latitude is placed in front of the storm (relative to its roughly westward propagation). Within the moist zone, the RH of all model grids from $900 \mathrm{hPa}$ to the model top of $20 \mathrm{hPa}$ are set to the maximum RH within the outer radius of the storm at each level by modifying specific humidity without changing temperature. In the intermediate moist front (MFI) simulation (Fig. 1c), the moist zone is located at the same place as for MF but the magnitude of the moisture perturbation is smaller ( $70 \%$ of the maximum RH at each level). Thus, the CTRL, MFI and MF cases represent the dry, intermediate moist and moist environments at the front of the storm, respectively.

In the moist rear (MR) simulation, a moist zone with the same area and magnitude of RH perturbations as in the MF run is placed to the south, roughly in the storm's rear quadrants (Fig. 1d). The dry rear (DR) simulation (Fig. 1e) is similar to the MR simulation but the magnitude of the $\mathrm{RH}$ perturbation is reduced to $30 \%$ of the maximum RH at each level, which is drier than the CTRL. So the dry, intermediate moist and moist environments at the rear of the storm are represented by the DR, CTRL and MR experiments, respectively.
Further sensitivity experiments with moisture zones of different sizes were also tested, and the results are not qualitatively sensitive to the choice of the areal extent of the moist zone. For brevity, only MF, MFI, MR and DR are discussed in addition to the CTRL. We also perform a set of simulations in which the vertical extent of the moisture perturbations in the MR configuration is varied to examine the vertical dependence of the environmental moisture impacts.

When a high-resolution model like WRF is initialized from global models, there is generally an adjustment period ("spin up") of about $12 \mathrm{~h}$. At $12 \mathrm{~h}$, the initial sharped-edged moisture perturbations have smoothed out to be representative of natural variability (Fig. 1f $-\mathrm{j}$ ). As shown in the results later (Figs. 2 and 4), the differences between the perturbed simulations and the CTRL experiment occur mostly after the $12 \mathrm{~h}$. Our discussions are thus focused on the simulations during $12-48 \mathrm{~h}$.

\subsection{Marsupial Paradigm}

The Marsupial Paradigm is a framework proposed by Dunkerton et al. (2009) to study the formation of a TC within tropical waves. Dunkerton et al. (2009) demonstrated that the critical layer of a tropical easterly wave is a region of approximately closed Lagrangian circulation (also called a "wave pouch"). The wave pouch protects the TC vortex from dry air intrusion to some extent, rendering a favorable environment for deep convection and TC formation. Owing to convergent flow, the wave pouch may have an opening that allows the influx of environmental air (see Fig. 3 in Wang et al., 2010). The Lagrangian boundary of the storm and its interaction with the ambient environment can be clearly illustrated by the streamlines in a frame of reference moving at the same speed with the wave (Fritz and Wang, 2013; Montgomery et al., 2010; Wang et al., 2009, 2012a, b). The 

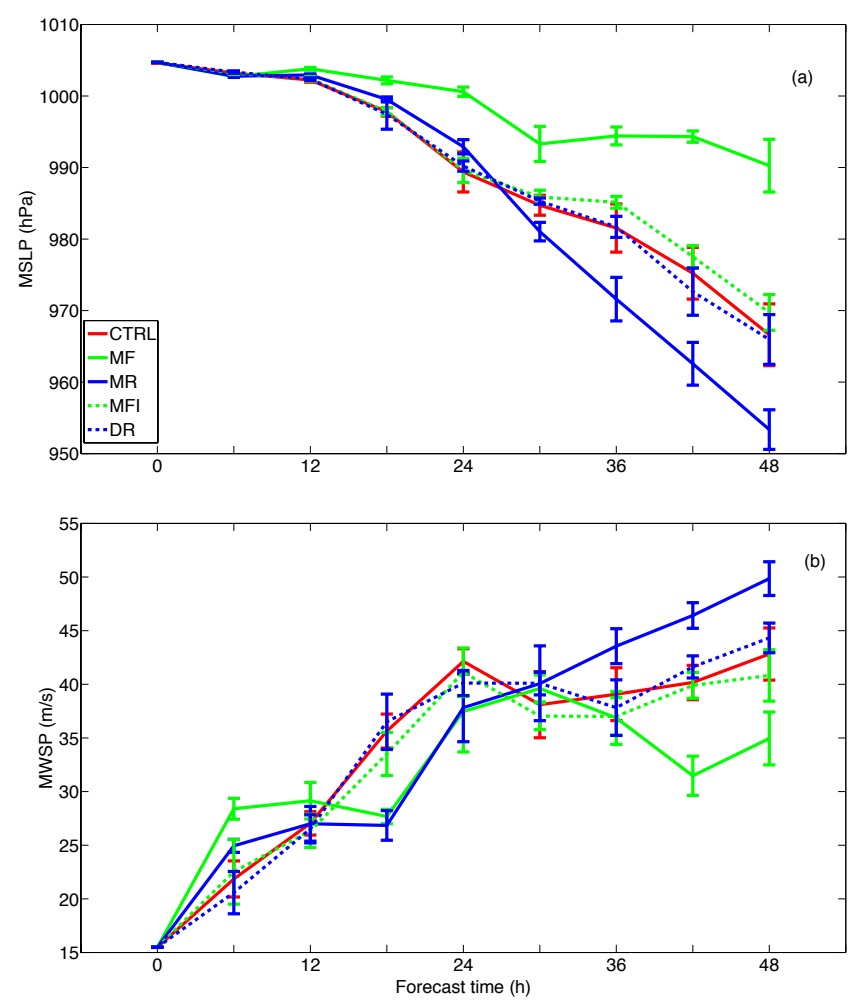

Figure 2. Time series of the model simulated ensemble mean and standard deviation of (a) MSLP (hPa) and (b) $\operatorname{MWSP}\left(\mathrm{m} \mathrm{s}^{-1}\right)$.

translated streamlines in a co-moving frame, which resemble the flow trajectories, provide a Lagrangian view of the storm evolution. Although the Marsupial Paradigm framework was proposed for TC formation, we adopt the concept in this study to investigate the impacts of asymmetric environmental moisture on TC intensification and structure. In the following analysis, the modeled streamlines are translated from the Earth-relative frame to the co-moving frame based on the estimated storm propagation speed from the automatic vortex-following algorithm in the WRF.

\section{Storm evolution in the control simulation}

As shown in Fig. 2, the simulated storm in the CTRL experiment (red lines) intensifies in the first $24 \mathrm{~h}$. During 24-30 h, the simulated MSLP shows a slowing down of the intensification (Fig. 2a) while the MWSP (Fig. 2b) exhibits a weakening trend. The storm continues its intensification in the following $18 \mathrm{~h}$. The MWSP of the simulated storm increases by $21 \mathrm{~m} \mathrm{~s}^{-1}$ from 6 to $48 \mathrm{~h}$ while the MSLP deepens by $38 \mathrm{hPa}$. The simulated intensification rate in the CTRL experiment is less than that for Hurricane Earl (2010). Since this study focuses on understanding the role of environmental moisture in TC intensification, the differences between the sensitivity experiments and the CTRL are of interest. The difference
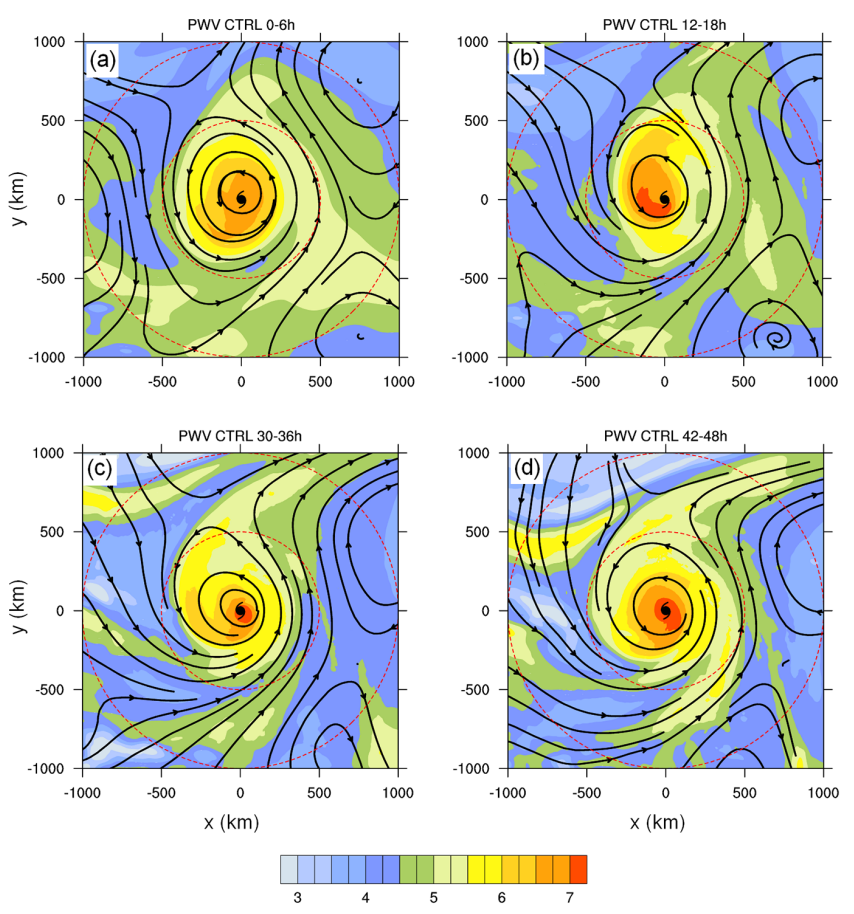

Figure 3. The mean translated streamline below $5 \mathrm{~km}$ and columnintegrated PWV $(\mathrm{cm})$ (shading) in the WRF CTRL simulation in the storm-following coordinate: (a) $0-6 \mathrm{~h}$; (b) $12-18 \mathrm{~h}$; (c) $30-36 \mathrm{~h}$; (d) 42-48 h. The hurricane symbol shows the TC center. The dashed red circles represent the radius of 500 and $1000 \mathrm{~km}$. All the data are taken from the outer model domain.

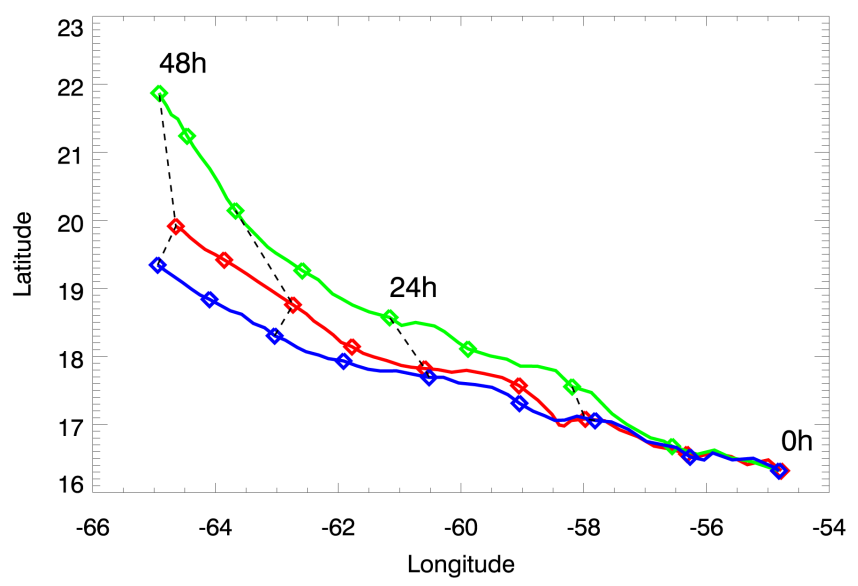

Figure 4. Storm tracks for CTRL (red), MF (green) and MR (blue). Every $6 \mathrm{~h}$ is identified with a diamond symbol. Black dashed lines connect storm position at the same forecast time for every $12 \mathrm{~h}$.

between the simulated storm in the CTRL experiment and observed Hurricane Earl is not a primary concern.

Figure 3 shows the PWV and translated streamlines of the WRF CTRL simulation in the co-moving frame. Averages over four periods $(0-6,12-18,30-36$ and $42-48 \mathrm{~h})$ are displayed. At the initial time (Fig. 3a), the storm core (indicated 
by the relative large PWV $>5 \mathrm{~cm}$ ) is collocated with the storm Lagrangian structure (indicated by the nearly enclosed streamlines). The storm Lagrangian structure is closed to the west of the storm, where dry air is located. Thus, there is a favorable environment for the intensification of the storm, as dry air intrusion would be limited and moisture in the vortex can be preserved. The inner region of the storm continues to moisten (Fig. 3b) as the storm intensifies in the first $24 \mathrm{~h}$ (Fig. 2), and the dry zone to the northwest of the storm becomes even drier (Fig. 3b). Contrarily, the moist region to the south and southeast of the storm diminishes in magnitude. The storm Lagrangian structure is open to the southwest at this time. In the next $24 \mathrm{~h}$ (Fig. $3 \mathrm{c}$ and d), the storm center keeps moistening while the dry air approaches the opening of the storm Lagrangian structure to the southwest of the storm.

\section{Impacts of environmental moisture}

\subsection{Summary of sensitivities in TC intensity and track}

Figure 2a shows the evolution of MSLP from four sensitivity experiments for comparison with the CTRL simulation. Except for the first $6 \mathrm{~h}$ of the $48 \mathrm{~h}$ integration, the MF experiment (with an ensemble mean of $990 \mathrm{hPa}$ at the $24 \mathrm{~h}$ simulation) has higher MSLP than the CTRL simulation (whose ensemble mean is $967 \mathrm{hPa}$ at that same time). The MR experiment produces comparable (or slightly higher) MSLP to the CTRL simulation in the first $24 \mathrm{~h}$. Afterwards, the storm in the MR experiment strengthens much faster than its CTRL counterpart. The MSLP in the MR simulation reaches $953 \mathrm{hPa}$ at the $48 \mathrm{~h}$ forecast, the lowest among all the experiments. Similar experiments with initialization at $12 \mathrm{~h}$ earlier show consistent results to the CTRL, MF and MR experiments, except a more intense storm developed in the experiment with a moist perturbation in the rear (figure not shown). Both the MFI and DR simulations have minor impacts on hurricane intensity, compared to the CTRL, throughout the $48 \mathrm{~h}$ integration.

Similar trends of storm evolution appear in the simulated MWSP (Fig. 2b). Both the MF and MR simulations produce a stronger storm at the $6 \mathrm{~h}$ forecast than the CTRL run. Between 18 and $24 \mathrm{~h}$, the strength of the storm is comparable between MF and MR but weaker than that in the CTRL. After $30 \mathrm{~h}$, the MF experiment produces a weaker storm relative to the CTRL simulation while the storm intensifies faster in the MR run. By the end of the simulation at $48 \mathrm{~h}$, the ensemble mean MWSP is $35 \mathrm{~m} \mathrm{~s}^{-1}$ for MF, $43 \mathrm{~m} \mathrm{~s}^{-1}$ for CTRL and $50 \mathrm{~m} \mathrm{~s}^{-1}$ for MR. Consistent with MSLP, both the MFI and DR experiments have no significant impacts on the magnitude of MWSP relative to the CTRL.

Regarding storm track (Fig. 4), the storm in the MF experiment moves further northwestward than the CTRL case. A significant track difference starts to show at $12 \mathrm{~h}$, corresponding to the change in the MSLP. In the first $24 \mathrm{~h}$, the track
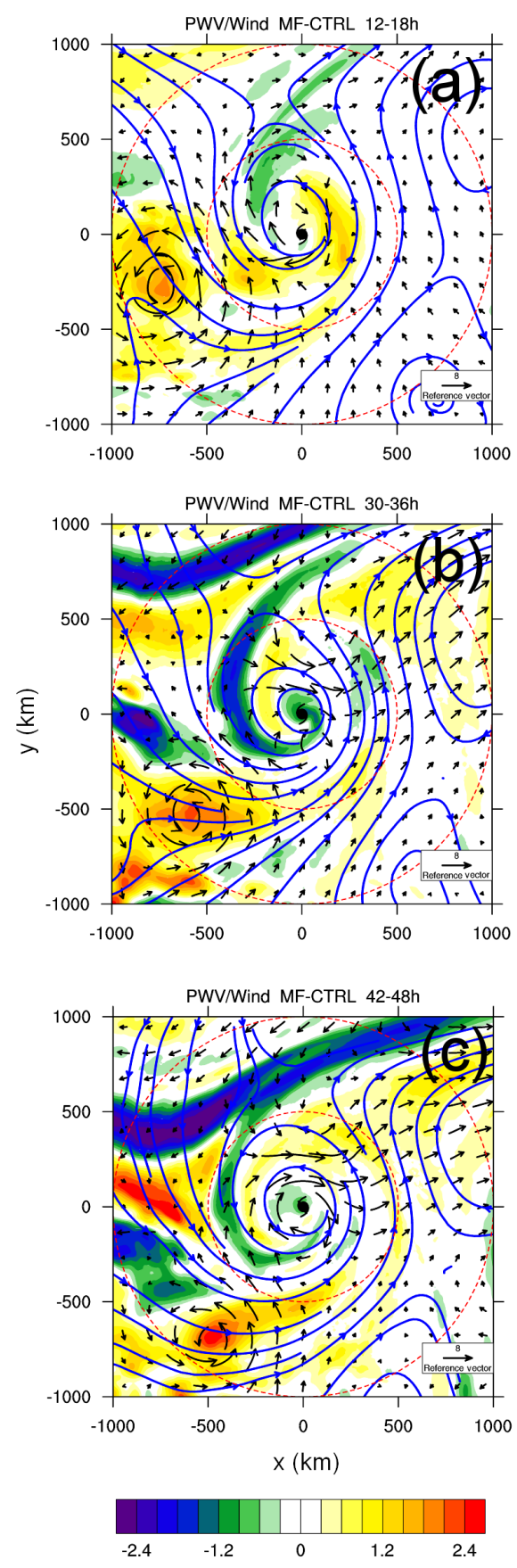

Figure 5. Differences of mean wind vector $\left(\mathrm{m} \mathrm{s}^{-1}\right)$ below $5 \mathrm{~km}$ and column-integrated PWV (cm) (shading) between the MF and CTRL simulations in the storm-following coordinate: (a) 12-18 h; (b) 30$36 \mathrm{~h}$; (c) $42-48 \mathrm{~h}$. The blue streamline is the translated streamline at the co-moving coordinate for the CTRL experiment at the corresponding time. The hurricane symbol shows the TC center. The dashed red circles represent the radius of $500 \mathrm{~km}$ and $1000 \mathrm{~km}$. All the data are taken from the outer model domain. Mean wind vectors and column-integrated PWV for CTRL and MF at each time are shown in Fig. S1 in the Supplement. 
differences are less than $110 \mathrm{~km}$. When the storm executes a gradual curve to the northwest, the track differences increase with a maximum difference of $220 \mathrm{~km}$ at $48 \mathrm{~h}$. In the last $24 \mathrm{~h}$, the significant deflection to the north with lower sea surface temperature may partly contribute to the weaker storm in the MF experiment. The MR experiment has relatively small changes in the storm track. In the last $24 \mathrm{~h}$, the storm in the MR experiment moves less northward comparing to the storm in the CTRL experiment, along with stronger intensification in the MR. The track differences are less than $70 \mathrm{~km}$ between MR and CTRL for all the $48 \mathrm{~h}$ integration. The track differences from the CTRL experiment are insignificant in the MFI and DR experiments (not shown).

Details of the storm evolution in each sensitivity experiment are investigated in a storm-following framework in the following subsections.

\subsection{MF experiment}

Figure 5 shows the differences of PWV and winds between the MF and CTRL experiments. At the initialization of the simulation (Fig. 1b), a nearly saturated region with a large amount of water vapor is prescribed to the west of the storm, where it is dry in the CTRL. The prescribed moist zone is outside of the storm Lagrangian boundary. In the following $18 \mathrm{~h}$, extensive precipitation (maximized between 6 and $12 \mathrm{~h}$; not shown) develops within the prescribed moist zone in the MF experiment (Fig. 6b), which is absent in the CTRL simulation (Fig. 6a). This supplemental convective activity induces a cyclonic circulation around the prescribed moist zone in the environment of the storm, resulting in a deformation of the storm Lagrangian structure with divergence to the west of the storm center (Fig. 5a).

Consequently, both moist air from the prescribed moist zone and dry air in the environment intrude into the storm vortex from the convective-deformed portion, leading to an asymmetric moisture structure (Fig. 5a-c) and diabatic heating fields (Figs. $6 \mathrm{~b}$ and 7). Dry environmental air has reached the storm inner core at 30-36 h (Fig. 5b). At $42-48 \mathrm{~h}$ forecast, a spiral band of convection with closed ring in the inner core forms in the CTRL case (Fig. 7d) while only a comma shape of convection is produced in the MF experiment (Fig. 7e) with much weaker storm intensity (Fig. 7f). In summary, convection in the environment in the MF case deforms the storm Lagrangian structure towards the dry front-side environment and facilitates the intrusion of dry air from the north into the inner core, creating asymmetric convection in the inner core and leading to the weakening of the storm (Nolan and Grasso, 2003; Nolan et al., 2007).

\subsection{MR experiment}

In the MR experiment, the prescribed moist zone is located in the already relatively moist environment to the south of the storm, outside of the storm Lagrangian boundary (Fig. 1c).
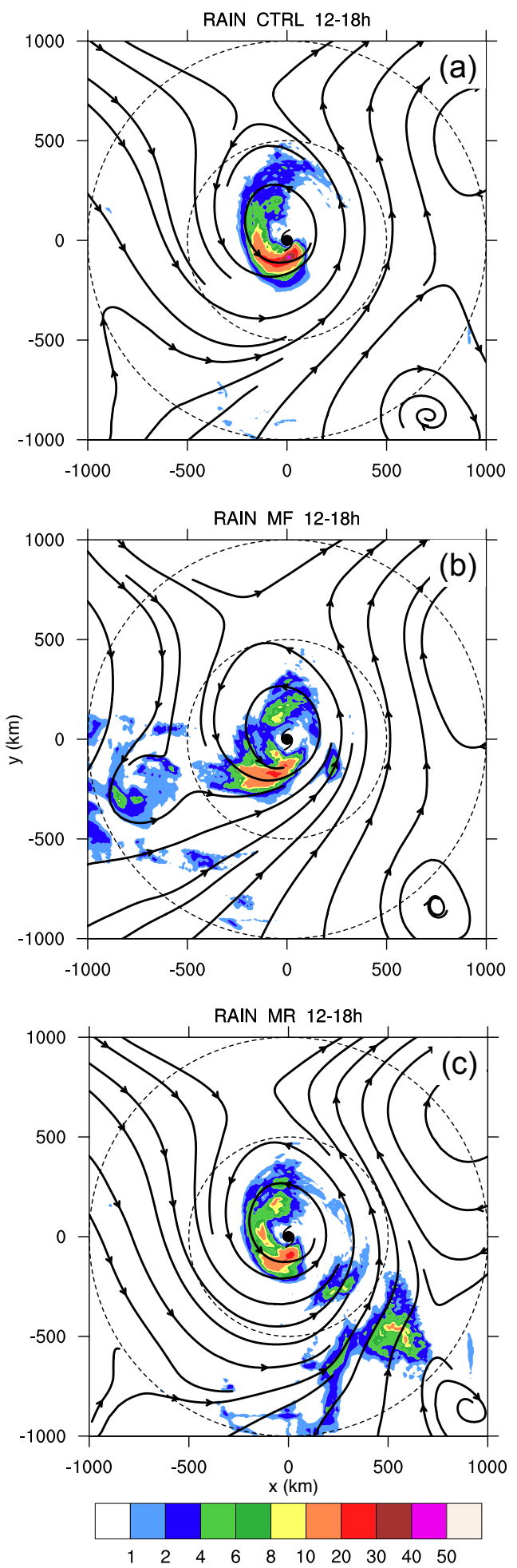

Figure 6. Mean rain rate $\left(\mathrm{mm} \mathrm{h}^{-1}\right)$ and streamlines below $5 \mathrm{~km}$ during $12-18 \mathrm{~h}$ in the storm-following coordinate: (a) CTRL; (b) MF; (c) MR. The hurricane symbol shows the TC center. The dashed black circles represent the radius of 500 and $1000 \mathrm{~km}$. All the data are taken from the outer model domain. 

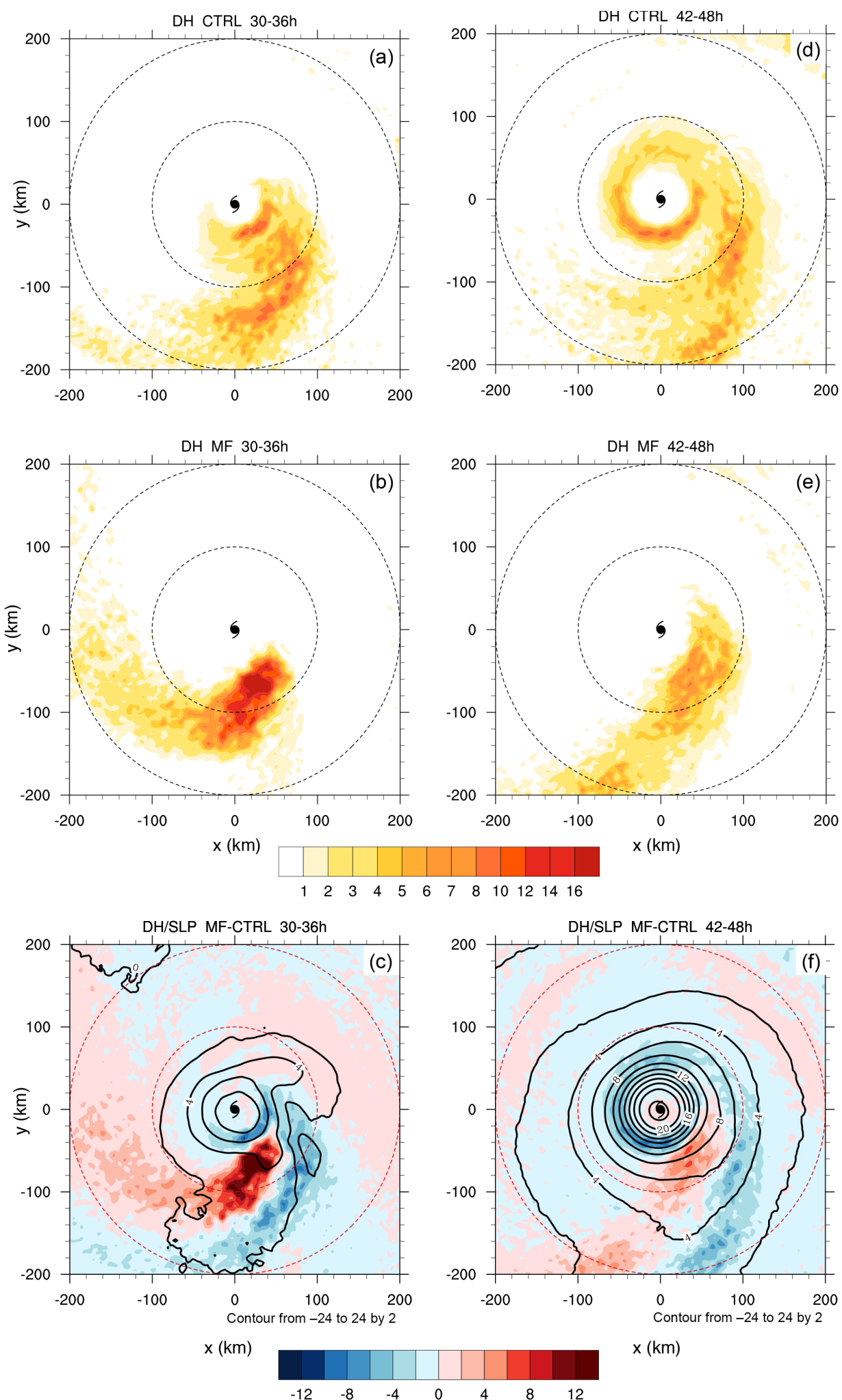

$\mathrm{x}(\mathrm{km})$

Figure 7. (a) Diabatic heating (DH; $\mathrm{K} \mathrm{day}^{-1}$ ) of CTRL in 30-36 h; (b) DH of MF in 30-36 h; (c) the difference of DH and SLP between MF and CTRL in 30-36 h; (d) DH of CTRL in 42-48 h; (e) DH of MF in 42-48 h; (f) the difference of DH and SLP between MF and CTRL in $42-48 \mathrm{~h}$ in the storm-following coordinate. The hurricane symbol shows the TC center. The dashed red circles represent the radius of 100 and $200 \mathrm{~km}$. All the data are taken from the inner model domain. 
Similar to the MF case, the nearly saturated moist perturbation induces convective activity and precipitation (Fig. 6c) beyond the storm vortex in the first $18 \mathrm{~h}$, resulting in a weaker storm compared to the CTRL case prior to $26 \mathrm{~h}$ (Fig. 2). Different from the MF case, the convection-induced deformation helps transport moisture to the east portions of the storm without an accompanying dry air intrusion (Fig. 8a).

Therefore, by 30-36h (Fig. 8b), more moisture appears within the core and also on the storm's north flank, where it is also moister than in the CTRL case (Fig. 3c). This results in a more symmetric storm, with better-defined spiral rainbands than the CTRL (Fig. 9a and b). Subsequently, the MR storm starts strengthening faster than the CTRL (Figs. 2 and 9c), and by the end of the $48 \mathrm{~h}$ integration, the convective activity of the inner core in the MR case (Fig. 9e) shows a nearly concentric ring without the long tail of the spiral band seen in the CTRL case (Fig. 9d). In summary, the convection in the environment enhances the inflow to the storm Lagrangian structure from the moist region and facilitates the moisture transport into the storm inner core in the MR case, leading to a more symmetric storm with higher intensity.

\subsection{MFI and DR experiments}

The MFI and DR experiments are similar to the MF and MR cases, respectively, except that their RH perturbation magnitude at each level is reduced in the prescribed zone. In both of the MFI and DR experiments (Fig. 10), the moisture perturbations do not promote convective activity in the environment of the storm. Throughout the $48 \mathrm{~h}$ integration, the storms in both the MFI and DR experiments contain the Lagrangian structures comparable to the CTRL case. The Lagrangian structure protects the storm well from intrusion of the environmental air. The prescribed moist air in the MFI and dry air in the DR wrap around the storm without entrainment into the storm vortex during the $48 \mathrm{~h}$ integration. There is no significant change in storm intensity and vortex structure of the MFI and DR experiments compared to the CTRL simulation. This is broadly consistent with Braun et al. (2012) that environment moisture content does not necessarily affect the storm intensity when the perturbation magnitude is not significant.

\subsection{Height dependency}

Another set of experiments are conducted to identify which layer of moisture is more important to promote TC intensification in the MR experiment. In these simulations, we limit the vertical extent of the moist perturbation to $900-500,900$ $300,850-500,500-300,500-20$ and 300-20 hPa, respectively. It is found that only the RH enhancements including the boundary layer (900-300 and $900-500 \mathrm{hPa}$ cases) promote significant intensification of the storm relative to the CTRL simulation (Fig. 11). When extra moisture is provided above $850 \mathrm{hPa}$, the intensity of the storm is quite similar to
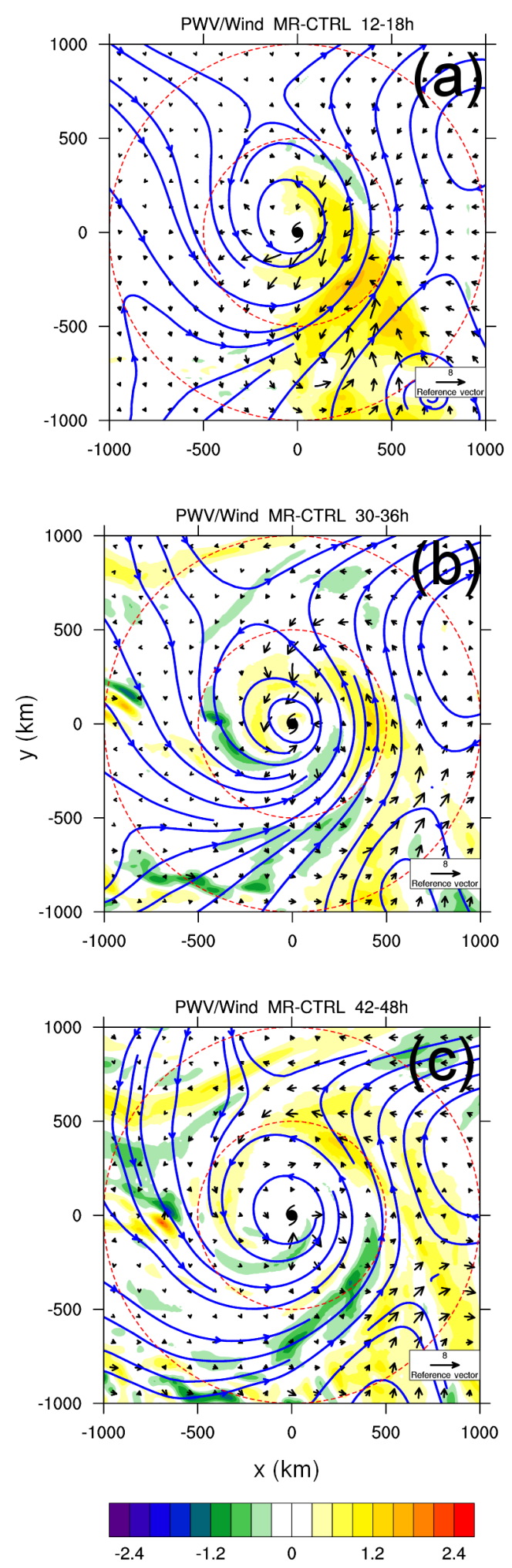

Figure 8. Same as Fig. 5 but for differences between the MR and CTRL experiments. Mean wind vectors and column-integrated PWV for CTRL and MR at each time are shown in Fig. S1 in the Supplement. 

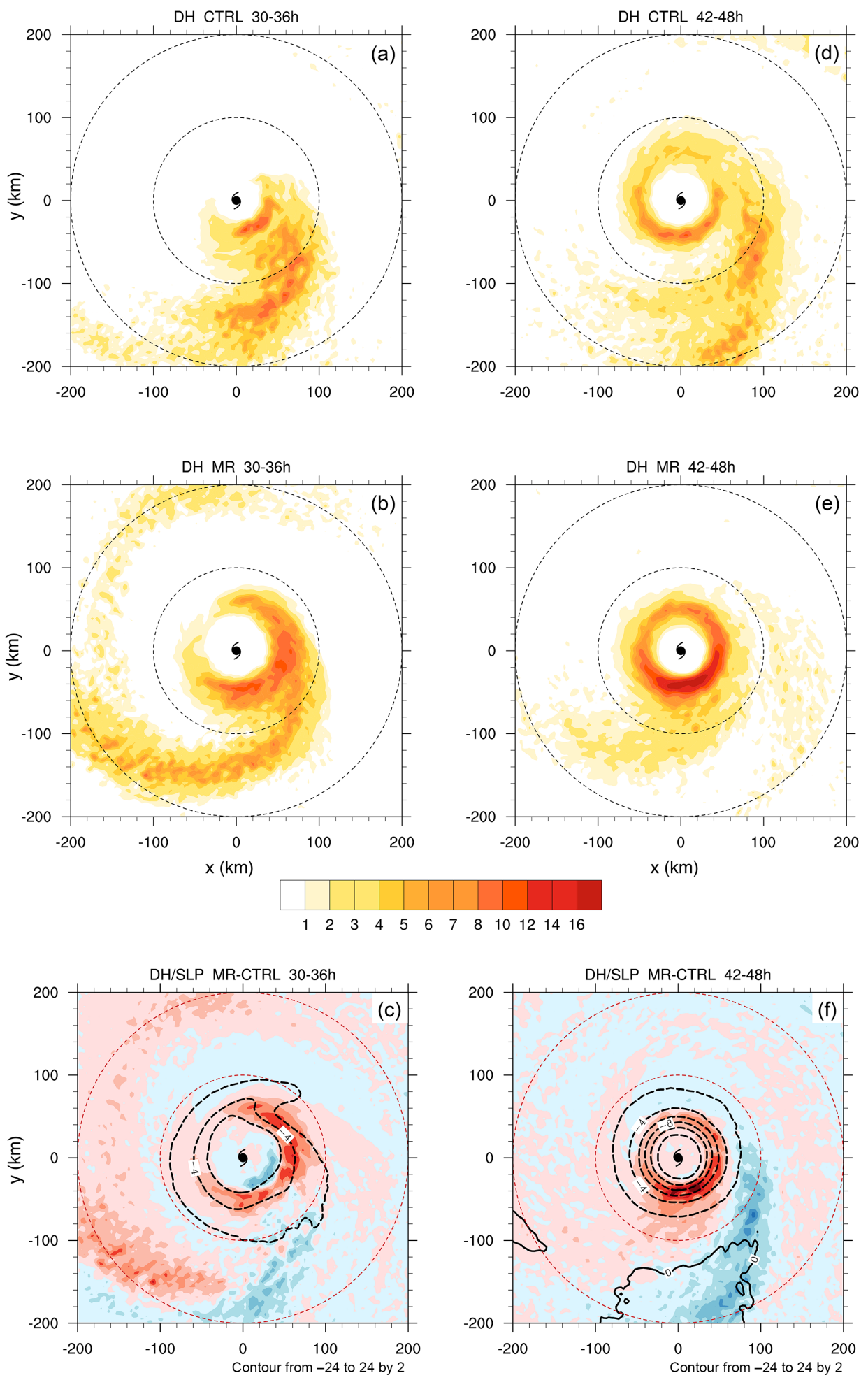

$x(\mathrm{~km})$

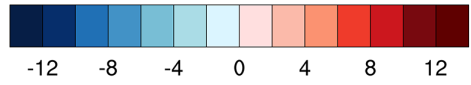

$x(\mathrm{~km})$

Figure 9. Same as Fig. 7 but for the MR and CTRL experiments. 

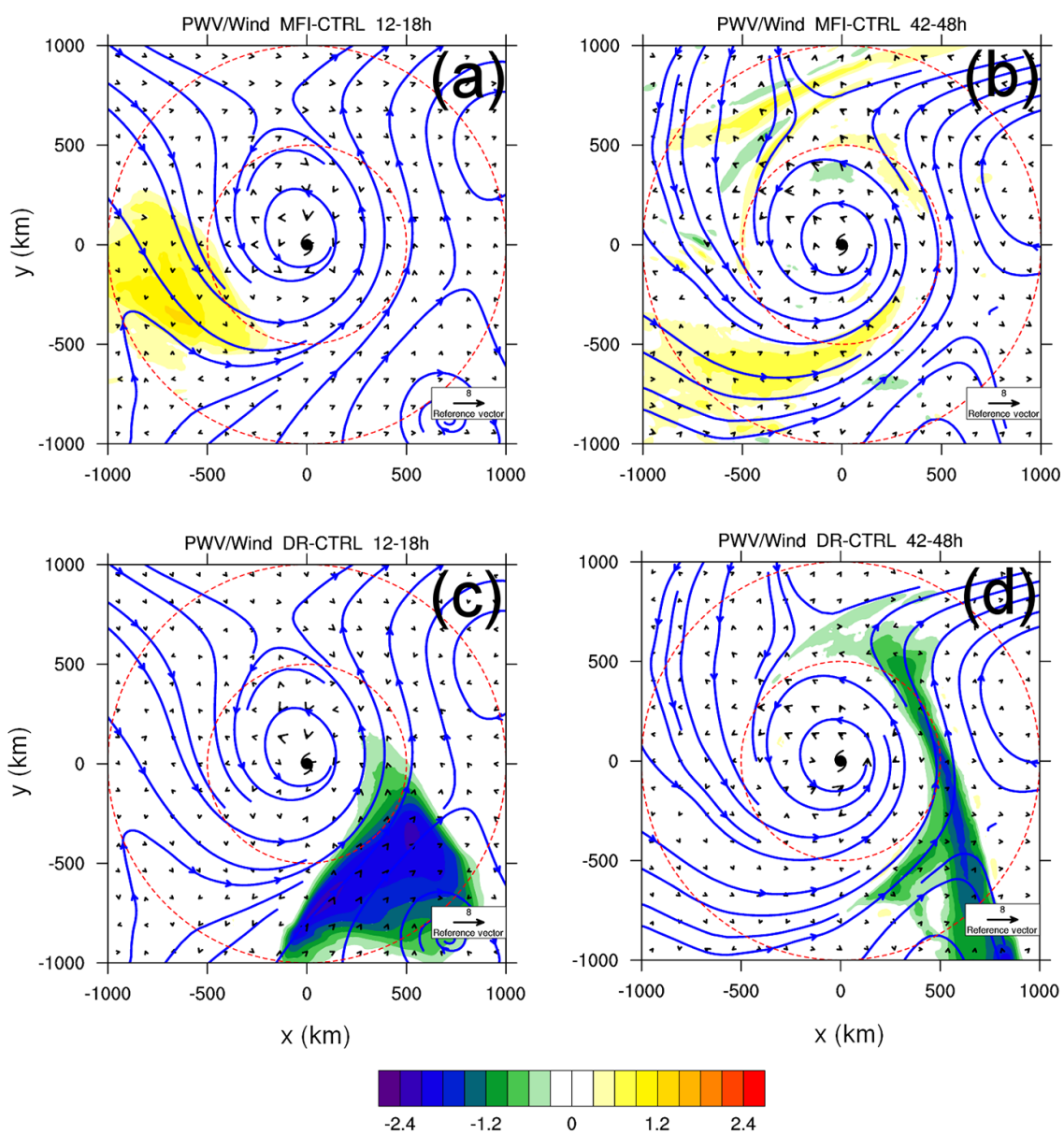

Figure 10. Difference of mean wind vector below $5 \mathrm{~km}$ and column-integrated PWV (cm) in the storm-following coordinate: (a) MFICTRL for 12-18 h; (b) MFI-CTRL for 42-48 h; (c) DR-CTRL for 12-18 h; (d) DR-CTRL for $42-48 \mathrm{~h}$. The blue streamline is the translated streamline at the co-moving coordinate for the CTRL case at corresponding time. The hurricane symbol shows the TC center. The dashed red circles represent the radius of 500 and $1000 \mathrm{~km}$. All the data are taken from the outer model domain.

the CTRL run or even slightly weaker than the CTRL case by the end of the simulations at $48 \mathrm{~h}$ integration, although convective activity induced by moisture perturbation is produced outside of the storm in some cases (for example, the $850-500 \mathrm{hPa}$ case). Note that saturation water vapor content in the boundary layer is significantly higher than in the middle and upper troposphere. Therefore, a small increase of $\mathrm{RH}$ in the boundary layer can provide much more moist static energy to fuel the storm intensification.

\section{Summary and discussion}

Guided by observations (Wu et al., 2012), the impacts of environmental moisture on TC intensity are examined in the WRF model, with a focus on the azimuthal asymmetry of moisture impacts. The Marsupial Paradigm framework is used to understand the evolution of the storm. The intensification process of a storm is simulated in the WRF CTRL simulation. When the moisture perturbation is not large enough to create additional convection outside of the storm, as in the MFI and DR experiments, the storm Lagrangian boundary serves as a barrier to protect the storm from intrusion of environmental air. No significant impact on the storm intensity and track is observed in the MFI and DR experiments.

However, when convective activity is promoted by the moisture perturbation and deforms the storm Lagrangian structure, as in the MF experiment, a storm that is weaker than the CTRL case occurs due to intrusion of dry environmental air from the northwest into the vortex through the convective-induced open Lagrangian structure, which leads to the asymmetry of convection in the storm inner core. The storm is also deflected to further northwest and approaches dry air, especially in the last $24 \mathrm{~h}$, which may also contribute to the weaker storm in the MF experiment. In contrast, convective deformation of the vortex in the MR experiment facilitates the entrainment of additional moisture from the south and results in more symmetric and powerful convection in the inner core with a higher intensity than the CTRL case. The 

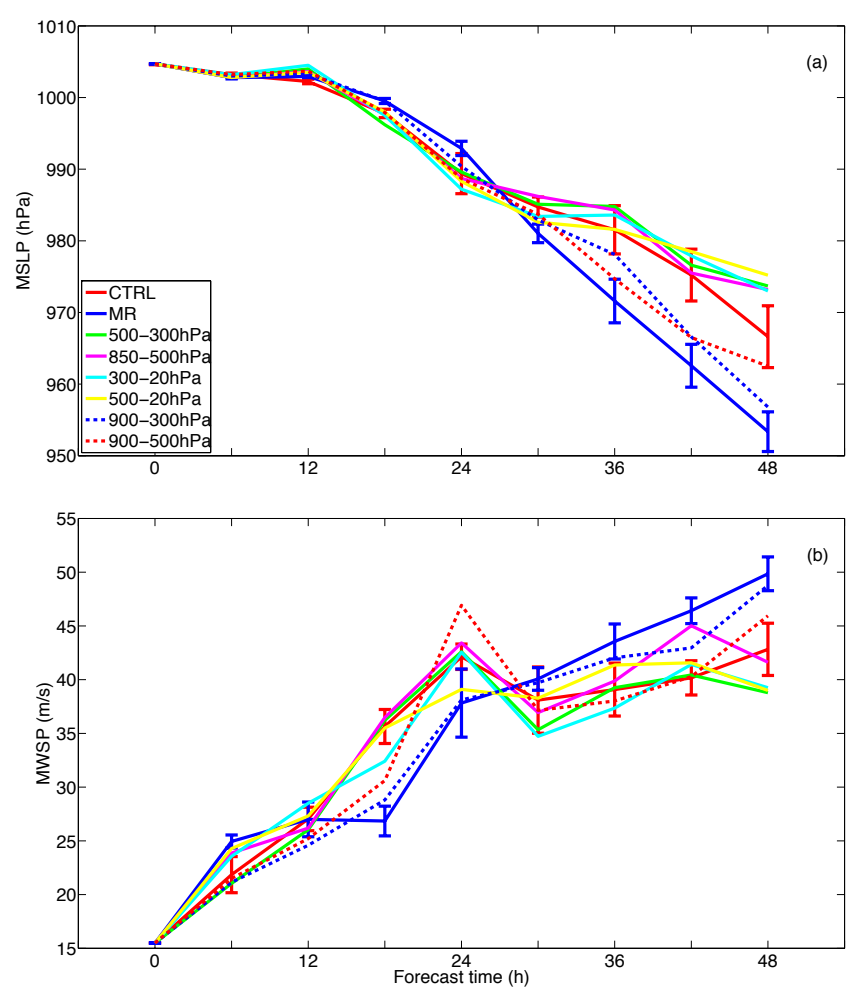

Figure 11. Time series of the model simulated (a) MSLP (hPa) and (b) MWSP $\left(\mathrm{m} \mathrm{s}^{-1}\right)$. CTRL in red; MR in blue; other simulations are the same as the MR run but with modification of moisture in differently prescribed pressure layer.

intensification is primarily contributed by enhanced moisture in the boundary layer. The distortion of the storm Lagrangian structure and changes in the moisture pathway play the key roles in the different response of the MF and MR cases.

This study demonstrates that the Marsupial Paradigm is a useful tool to study the interaction of a TC vortex with its environment at any stage of the storm development, not only limited to TC formation. Dunkerton et al. (2009) proposed that a closed circulation is favorable for TC formation. This study hypothesized an open storm Lagrangian structure can also benefit TC formation and intensification as long as the opening is towards a favorable environment (e.g., moist air).

The series of sensitivity experiments with different magnitudes and positions of moisture perturbations provide a comprehensive view of the interactions of environmental moisture with a tropical cyclone. Based on these results and previous studies (Braun et al., 2012; Ge et al., 2013; Hill and Lackmann, 2009; Kimball, 2006; Tao and Zhang, 2014; Wang, 2009; Ying and Zhang, 2012), we conclude that environmental moisture has limited impacts on storm intensity when it does not enter the storm vortex, similar to the insignificant impacts of dry air beyond $270 \mathrm{~km}$ noted in Braun et al. (2012). If the moisture enhancement produces enhanced convective activity within the vortex, however, the direct and indirect impacts on the storm can be complex. By itself, enhanced outer rainband activity (the direct effect) may weaken the storm (Wang, 2009; Ying and Zhang, 2012). Yet, the convective activity could also deform the storm vortex, more indirectly leading to changes in the nature of the moisture inflow. Consistent with conventional understanding, a dry air intrusion into the inner core that might opportunistically cause a vortex deformation (as in the MF case) and suppress the storm, while an enhanced moisture supply into the inner core (as in the MR case) promotes intensification of the storm. The disparate responses of TC intensity to moisture perturbations in the literature may largely be a result of the different magnitudes and relative locations of moisture perturbations to the storm vortex and, thus, their different abilities to deform the storm vortex.

This study demonstrates that storm structure is critical for understanding environmental impacts on TCs. Previous composite data analyses have been sampled with respect to the distance from the storm center, without consideration on the storm (structure). Most modeling studies prescribed moisture perturbations but did not pay much attention to their relative locations to the storm vortex. As shown in this study and previous papers, TCs respond differently to moisture perturbations in different locations (the inner core, the outer rainband region and the more distant environment). Thus, in order to better quantify moisture impacts on TCs, it is necessary to distinguish moisture in the outer rainband and moisture in the inner core of the storm as well as different environmental moisture distributions.

This study also explains, to some degree, the observational results by Shu and Wu (2006) that the dry SAL may have favorable or unfavorable impacts on TC intensification, depending on its position. Considering that the TCs in the North Atlantic usually have moisture inflow from the southern quadrants, when the SAL is located to the northwest of TCs, it may not affect the storm intensity, or may even indirectly favor TC intensification by suppressing the formation of convective rainbands outside of the storm. When dry air is located to the southeast or southwest of the TCs, however, the dry air may be entrained into the storm, leading to a weakening effect. The MF and MR experiments suggest that the "dry front and moist rear" distribution of environmental moisture is a favorable condition for TC intensification, consistent with the observational study of $\mathrm{Wu}$ et al. (2012). Given that environmental moisture can have different impacts on TCs once it enters into the storm, accurate characterizations of environmental moisture are important to TC intensity forecasts.

This study shows that convection in the environment can have either favorable or unfavorable impacts on the storm intensity. Thus, a better understanding of the interaction of the storm with environmental convective activity (e.g., trough interaction with storm) is also critical to improving TC intensity forecasts. 


\section{The Supplement related to this article is available online at doi:10.5194/acp-15-14041-2015-supplement.}

Acknowledgements. The work is conducted at the Jet Propulsion Laboratory, California Institute of Technology, under contract with NASA. The authors thank the funding support from the NASA Hurricane Science Research Program. Wang was supported by National Science Foundation Grant AGS-1118429. Helpful comments from Mark Boothe, Shuyi Chen and two anonymous reviewers are appreciated.

Edited by: F. Yu

\section{References}

Braun, S. A., Sippel, J. A., and Nolan, D. S.: The Impact of Dry Midlevel Air on Hurricane Intensity in Idealized Simulations with No Mean Flow, J. Atmos. Sci., 69, 236-257, doi:10.1175/JASD-10-05007.1, 2012.

DeMaria, M., Knaff, J. A., and Sampson, C.: Evaluation of longterm trends in tropical cyclone intensity forecasts, Meteor. Atmos. Phys., 97, 19-28, 2007.

Dunkerton, T. J., Montgomery, M. T., and Wang, Z.: Tropical cyclogenesis in a tropical wave critical layer: easterly waves, Atmos. Chem. Phys., 9, 5587-5646, doi:10.5194/acp-9-5587-2009, 2009.

Emanuel, K., DesAutels, C., Holloway, C., and Korty, R.: Environmental control of tropical cyclone intensity, J. Atmos. Sci., 61, 843-858, doi:10.1175/15200469(2004)061<0843:ECOTCI>2.0.CO;2, 2004.

Emanuel, K. A.: Dynamical theories of tropical convection, Aust. Meteor. Mag., 37, 3-10, 1989.

Fritz, C. and Wang, Z.: A Numerical Study of the Impacts of Dry Air on Tropical Cyclone Formation: A Development Case and a Nondevelopment Case, J. Atmos. Sci., 70, 91-111, doi:10.1175/JAS-D-12-018.1, 2013.

Ge, X., Li, T., and Peng, M.: Effects of Vertical Shears and Midlevel Dry Air on Tropical Cyclone Developments, J. Atmos. Sci., 70, 3859-3875, doi:10.1175/JAS-D-13-066.1, 2013.

Hendricks, E. A., Peng, M. S., Fu, B., and Li, T.: Quantifying Environmental Control on Tropical Cyclone Intensity Change, Mon. Weather Rev., 138, 3243-3271, doi:10.1175/2010MWR3185.1, 2010.

Hill, K. A. and Lackmann, G. M.: Influence of Environmental Humidity on Tropical Cyclone Size, Mon. Weather Rev., 137, 32943315, doi:10.1175/2009MWR2679.1, 2009.

Hong, S.-Y., Noh, Y., and Dudhia, J.: A New Vertical Diffusion Package with an Explicit Treatment of Entrainment Processes, Mon. Weather Rev., 134, 2318-2341, doi:10.1175/MWR3199.1, 2006.

Iacono, M. J., Delamere, J. S., Mlawer, E. J., Shephard, M. W., Clough, S. A., and Collins, W. D.: Radiative forcing by long-lived greenhouse gases: Calculations with the AER radiative transfer models, J. Geophys. Res., 113, D13103, doi:10.1029/2008JD009944, 2008.
Kain, J. S.: The Kain-Fritsch Convective Parameterization: An Update, J. Appl. Meteor., 43, 170-181, doi:10.1175/15200450(2004)043<0170:TKCPAU>2.0.CO;2, 2004.

Kaplan, J. and DeMaria, M.: Large-scale characteristics of rapidly intensifying tropical cyclones in the North Atlantic basin, Weather Forecast., 18, 1093-1108, 2003.

Kaplan, J., DeMaria, M., Knaff, J. A.: A Revised Tropical Cyclone Rapid Intensification Index for the Atlantic and Eastern North Pacific Basins, Weather Forecast., 25, 220-241, doi:10.1175/2009WAF2222280.1, 2010.

Kimball, S. K.: A Modeling Study of Hurricane Landfall in a Dry Environment, Mon. Weather Rev., 134, 1901-1918, doi:10.1175/MWR3155.1, 2006.

Montgomery, M. T., Wang, Z., and Dunkerton, T. J.: Coarse, intermediate and high resolution numerical simulations of the transition of a tropical wave critical layer to a tropical storm, Atmos. Chem. Phys., 10, 10803-10827, doi:10.5194/acp-1010803-2010, 2010.

Nolan, D. S. and Grasso, L. D.: Nonhydrostatic, ThreeDimensional Perturbations to Balanced, Hurricane-Like Vortices. Part II: Symmetric Response and Nonlinear Simulations, J. Atmos. Sci., 60, 2717-2745, doi:10.1175/15200469(2003)060<2717:NTPTBH>2.0.CO;2, 2003.

Nolan, D. S., Moon, Y., and Stern, D. P.: Tropical Cyclone Intensification from Asymmetric Convection: Energetics and Efficiency, J. Atmos. Sci., 64, 3377-3405, doi:10.1175/JAS3988.1, 2007.

Skamarock, W. C., Klemp, J. B., Dudhia, J., Gill, D. O., Barker, D. M., Wang, W., and Powers, J. G.: A Description of the Advanced Research WRF Version 3, NCAR Technical Note TN-468+STR, 113 pp., 2008.

Shu, S. and Wu, L.: Analysis of the influence of Saharan air layer on tropical cyclone intensity using AIRS/Aqua data, Geophys. Res. Lett., 36, L09809, doi:10.1029/2009GL037634, 2009.

Tang, B. and Emanuel, K.: Sensitivity of tropical cyclone intensity to ventilation in an axisymmetric model, J. Atmos. Sci., 69, 2394-2413, 2012.

Tao, D. and Zhang, F.: Effect of environmental shear, seasurface temperature, and ambient moisture on the formation and predictability of tropical cyclones: An ensemblemean perspective, J. Adv. Model. Earth Syst., 6, 384-404, doi:10.1002/2014MS000314, 2014.

Thompson, G., Field, P. R., Rasmussen, R. M., and Hall, W. D.: Explicit Forecasts of Winter Precipitation Using an Improved Bulk Microphysics Scheme. Part II: Implementation of a New Snow Parameterization, Mon. Weather Rev., 136, 5095-5115, doi:10.1175/2008MWR2387.1, 2008.

Wang, Y.: How Do Outer Spiral Rainbands Affect Tropical Cyclone Structure and Intensity?, J. Atmos. Sci., 66, 1250-1273, doi:10.1175/2008JAS2737.1, 2009.

Wang, Z., Montgomery, M. T., and Dunkerton, T. J.: A dynamicallybased method for forecasting tropical cyclogenesis location in the Atlantic sector using global model products, Geophys. Res. Lett., 36, L03801, doi:10.1029/2008GL035586, 2009.

Wang, Z., Montgomery, M. T., and Dunkerton, T. J.: Genesis of Pre-hurricane Felix (2007). Part I: The Role of the Wave Critical Layer, J. Atmos. Sci., 67, 1711-1729, 2010. 
Wang, Z., Montgomery, M. T., and Fritz, C.: A First Look at the Structure of the Wave Pouch during the 2009 PREDICT-GRIP Dry Runs over the Atlantic, Mon. Weather Rev., 140, 1144-1163, doi:10.1175/MWR-D-10-05063.1, 2012a.

Wang, Z., Dunkerton, T. J., and Montgomery, M. T.: Application of the Marsupial Paradigm to Tropical Cyclone Formation from Northwestward-Propagating Disturbances, Mon. Weather Rev., 140, 66-76, doi:10.1175/2011MWR3604.1, 2012b.
Wu, L., Su, H., Fovell, R. G., Wang, B., Shen, J. T., Kahn, B. H., Hristova-Veleva, S. M., Lambrigtsen, B. H., Fetzer, E. J., and Jiang, J. H.: Relationship of environmental relative humidity with North Atlantic tropical cyclone intensity and intensification rate, Geophys. Res. Lett., 39, L20809, doi:10.1029/2012GL053546, 2012.

Ying, Y. and Zhang, Q.: A Modeling Study on Tropical Cyclone Structural Changes in Response to Ambient Moisture Variations, J. Meteorol. Soc. Jpn., 90, 755-770, doi:10.2151/jmsj.2012-512, 2012. 